\title{
The value of urine specimens in screening for male urethritis and its microbial aetiologies in Tanzania
}

P Mayaud, J Changalucha, H Grosskurth, G Ka-Gina, J Rugemalila, J Nduba, J Newell, R Hayes, D Mabey

Abstract

Objective-To evaluate the first void urine (FVU) specimen in screening for urethritis and its microbial aetiologies in a male African population in which urinary schistosomiasis is also prevalent.

Patients and Methods-Two hundred and forty eight males aged 15-54 years provided FVU specimens: 55 patients from a clinic for sexually transmitted diseases (STD), 151 patients from a medical outpatient clinic and 42 villagers from an area of high endemicity for $S$ haematobium. Specimens were tested for leucocyte esterase (LE) using a dipstick (NephurTest + Leuco, Boehringer-Mannheim France SA). Ova of $S$ haematobium were sought in terminal urine samples from all subjects. For all STD patients, and all medical outpatients with a positive LE test, urine and urethral swabs were tested for Chlamydia trachomatis antigen, and urethral swabs were tested for Neisseria gonorrhoeae by gram stain and isolation.

Results-The prevalence of LE positivity was $38 / 41$ in STD patients with urethral signs or symptoms (93\%), 5/14 among other STD patients $(36 \%), 21 / 151$ among medical outpatients (15\%) and $13 / 42$ among villagers (31\%). As a screening test for urethral infection (detection of gonorrhoea or chlamydia and/or $\geqslant 5$ polymorphs per high power field on gram stain) the LE test had a sensitivity of $94 \%$ and a specificity of $53 \%$ among STD patients. Of 24 STD patients with gonococcal or chlamydial infection, 23 had a positive LE test $(96 \%)$. Among general medical outpatients, 12 of 22 with a positive LE test had either conventionally defined urethritis or gonococcal or chlamydial infection, giving a positive predictive value of $55 \%$ for the LE test in this group. Of 18 subjects in all groups with urinary schistosomiasis nine had a positive LE test (50\%), although three of these also had gonorrhoea. Chlamydial antigen was detected in the FVU specimen of all six subjects in whom it was detected in a urethral swab, and in an additional three subjects in the outpatient group.

Conclusions-The FVU, which is an easily collected and non-invasive specimen, can provide valuable information on the prevalence of urethritis and on its microbial aetiology among the general male population in African countries.
(Genitourin Med 1992;68:361-365)

\section{Introduction}

The rapid heterosexual spread of the human immunodeficiency virus (HIV) in Africa is alarming, with the seroprevalence in the general adult population now exceeding $10 \%$ in many cities of East and Central Africa. ${ }^{1-3}$

The role of other sexually transmitted diseases (STDs) as potential cofactors for the transmission of HIV is increasingly recognised. ${ }^{4-9}$ There is evidence that the infectivity of an HIV-infected person, and/or the susceptibility of an HIV-uninfected person, may be increased in the presence of certain STDs. Plausible biological mechanisms support this idea. Genital ulcer diseases (GUD) may disrupt genital epithelial integrity, providing a portal for entry or exit of the virus. Many STDs produce an inflammatory response, which may increase the number of infected or target cells in the genital tract. ${ }^{56}$

Since STDs are highly prevalent in many African populations, ${ }^{9-13}$ STD control programmes may play an important role in slowing the spread of the HIV epidemic. ${ }^{78}$ The impact of such programmes in the general population will depend upon: the magnitude of the causal link between STDs and transmission of HIV; the proportion of HIV infections attributable to STDs, which, in turn depends on the prevalence and distribution of STDs in the general population; and the effectiveness of the programme in reducing the prevalence of STDs.

Most published studies have measured the prevalence of STDs in urban or high risk populations. ${ }^{4-6}$ In order to estimate their prevalence in the general population, and to evaluate the impact of control programmes, there is a need to develop and test screening methods that are appropriate for lower risk populations.

The leucocyte esterase test (LE test) is a simple, cheap and non-invasive screening method for detecting urethritis among males which has been evaluated in different settings in Europe and the USA. ${ }^{14-18}$ To our knowledge there are no published data on the evaluation of the LE test in an African population and the test has never been utilised for epidemiological studies on general populations.

The primary objective of this study was to determine the value of first void urine specimens (FVU) in screening for male urethral infection (gonorrhoea, chlamydial infection or non-specific urethritis) using the LE dipstick test in an African setting. Since urianry schis- 
tosomiasis was prevalent in the study population, further evaluation of the interference of this parasitosis with the dipstick result was also warranted. We have also evaluated the FVU for the diagnosis of urethral chlamydial infection by antigen detection in this population.

\section{Patients and methods}

Study populations

This study, performed in July 1991, was carried out at two sites in Mwanza Region, north-western Tanzania: at Sekou Toure Hospital, which is the main health facility run by the Municipal Council of Mwanza, and in the village of Buhongwa.

At the hospital, eligible subjects were male patients aged 15-54 years either attending the Medical Outpatient Department or referred to the STD clinic. In all 214 patients were eligible, comprising 55 from the STD clinic and 159 from Medical Outpatients. One subject from the Medical Outpatient Department refused to participate, and a further seven were excluded from the analysis because of incomplete data.

In Buhongwa, a village highly endemic for $S$ haematobium, a random sample of the population aged 15-54 years was enrolled to investigate the effects of $S$ haematobium on the dipstick results. Only the results of the 42 eligible male subjects are reported here.

Twenty to $25 \mathrm{ml}$ of a first catch urine was collected from all study subjects into a sterile container. Subjects had not passed urine for at least two hours (median time was four hours). All STD patients had a cotton wool tipped charcoal swab inserted $1-2 \mathrm{~cm}$ inside the urethra, withdrawn, smeared onto a glass slide for Gram stain (polymorph (PMN) count and presence of gram negative intracellular diplococci) and then placed into a container with Stuart's transport medium. The specimen was kept at room temperature until the end of the clinic, when it was transported to the laboratory. A second cotton wool tipped aluminium swab was inserted $3-4 \mathrm{~cm}$ into the urethra, gently rotated, withdrawn and then placed in a cryotube containing chlamydia transport medium (NovoBiolabs, UK). The container was immediately refrigerated at $+4^{\circ} \mathrm{C}$.

Subjects attending the outpatient clinic who were noted to have a positive LE test had two urethral swabs taken in the same way as STD patients.

All patients in whom a STD was diagnosed were treated according to guidelines issued by the Tanzanian Ministry of Health. All those in whom schistosomiasis was diagnosed were treated with a single dose of praziquantel $40 \mathrm{mg} / \mathrm{kg}$.

\section{Laboratory methods}

Urine specimens were thoroughly mixed prior to testing by the LE dipstick (Nephur-Test + Leuco, Boehringer-Mannheim France SA) according to the manufacturer's instructions; 60 to 120 seconds after immersion the colour reaction was compared with a standardised colour chart. The erythrocyte test on the same dipstick was used to detect the presence of red blood cells or haemoglobin.

After dipstick testing urine samples were divided into two equal aliquots. The first was centrifuged for 5 minutes at $2700 \mathrm{rpm}$; the resuspended pellet was immediately examined under the $\times 10$ objective by an experienced technician for the diagnosis of schistosomiasis (qualitative method, that is presence or absence of ova of $S$ haematobium). The second aliquot was centrifuged for 30 minutes at $4000 \mathrm{~g}$. The supernatant was discarded and $1 \mathrm{ml}$ of IDEIA specimen diluent buffer added to the pellet. The specimen was kept at $+4^{\circ} \mathrm{C}$ for a maximum of 6 days.

All urine specimens from STD patients, and those from outpatients with a positive LE test, were tested for chlamydial antigen using a genus specific antigen detecting immunoassay (IDEIA, NovoBiolabs, UK), according to the manufacturer's instructions. Urethral swabs were also tested for chlamydial antigen in this way. Optical densities were read using an ELISA plate-reader and results were expressed as positive, negative or grey-zone.

Urethral swabs were removed from Stuart's medium, inoculated on to a modified New York City medium and incubated at $37^{\circ} \mathrm{C}$ in $5 \% \mathrm{CO} 2$ for 48 hours. $N$ gonorrhoeae was identified by colonial morphology, gram stain and positive oxidase and catalase tests.

\section{Definition of "Urethral Infection"}

Since the primary aim of this study was to evaluate the LE test in screening for the presence of sexually transmitted urethral infections, we have defined urethral infection as the presence of 5 or more PMNs per high power field on Gram stain of a urethral smear and/or the presence of $N$ gonorrhoeae (detected by culture) or $C$ trachomatis antigen. In this study, as in others, not all subjects with gonococcal or chlamydial infection of the urethra had urethritis as defined conventionally in terms of PMNs on the urethral smear.

\section{Results}

STD clinic patients

Of 55 STD patients $32(58 \%)$ presented with a urethral discharge, nine (16\%) complained of urethral symptoms without evidence of discharge, and 11 of the remaining 14 had GUD alone. Ten patients (18\%) presented with both GUD and urethral signs or symptoms.

The prevalence of urethral infection was $36 / 55$ (65\%) among STD clinic patients (table 1). Twenty four patients $(44 \%)$ had urethral infection caused by $N$ gonorrhoeae and/or $C$ trachomatis: twenty two (40\%) had $N$ gonorrhoeae and four $(7 \%)$ had $C$ trachomatis, two patients having a dual infection. Twelve patients $(22 \%)$ had non-specific urethritis (NSU) (PMN $\geqslant 5$ on Gram stained smear in the absence of chlamydial antigen or a positive culture for $N$ gonorrhoeae).

The prevalence of gonorrhoea among patients with urethral syndrome (urethral discharge and/or dysuria) was $22 / 41$ (54\%). The 
Table 1 Performance of leucocyte esterase test among 55 male STD patients

\begin{tabular}{lrrrl}
\hline STD Category & $N$ & $L E+$ & $P M N \geqslant 5$ & Schisto +ve \\
\hline Urethral infection & 36 & 34 & 31 & 2 \\
NG + and/or CT + & 24 & 23 & 19 & 2 \\
NSU & 12 & 11 & 12 & 0 \\
Urethral syndrome +/ no infection detected & 8 & 6 & 0 & 0 \\
Other + & 11 & 3 & 0 & 0 \\
Total & 55 & 43 & 31 & 2 \\
\hline
\end{tabular}

${ }^{\star}$ NSU $=$ Non-specific urethritis. Diagnosed if NG - and CT - , but PMN $\geqslant 5$.

+ Patient complaining of dysuria and/or discharge; or clinical evidence of discharge.

†Including 9 with genital ulcer, $1 \mathrm{LGV}, 1$ scabies.

prevalence of chlamydial infection in this group was $4 / 41(10 \%)$. 9/41 (22\%) were suffering from NSU, whilst no aetiology or evidence of urethritis was found in $8(18 \%)$. NSU was detected in an additional three patients who had no urethral signs or symptoms.

Value of the LE test in screening for urethral infection

Forty three patients (78\%) had FVU specimens with a positive LE test (table 1). The sensitivity of the LE test in detecting urethral infection was $94 \%$, and the specificity $53 \%$. When the test was evaluated for detecting the presence of either $N$ gonorrhoeae or $C$ trachomatis, the sensitivity was $96 \%$. In all, the LE test missed only one case of positive gonococcal culture, which would also have been missed by the PMN count; and it missed one NSU case. By comparison the PMN count failed to detect four cases of gonorrhoeae and one of chlamydial infection. The sensitivity of the PMN count for the detection of either organism was thus $79 \%$.

FVU for the diagnosis of chlamydial infection All patients testing positive for IDEIA on urethral swab were also positive on FVU specimen, but an additional patient had a "grey-zone" result for his urine specimen. Unfortunately this was not evaluated further. Specimens were retained for microimmunofluorescence testing but they did not survive the journey from Mwanza to London.

\section{Outpatient group}

The categories of complaints of these patients are displayed in table 2 . Thirteen patients $(9 \%)$ presenting with "dysuria" in this group had not been referred to the STD clinic by the clinical officer who had examined them and who had believed that they were not STDrelated.

Table 2 Leucocyte esterase test and urethral infections in 151 outpatients

\begin{tabular}{|c|c|c|c|c|c|c|}
\hline \multirow[b]{2}{*}{ Outpatient Group } & \multirow[b]{2}{*}{$N$} & \multirow[b]{2}{*}{$L E+$} & \multicolumn{3}{|c|}{ Urethral findings in $L E+$ patients } & \multirow[b]{2}{*}{ Schisto + ve } \\
\hline & & & Urethra infection & $N G+/ C T+$ & $N S U$ & \\
\hline Dysuria & 13 & 4 & 4 & 2 & 2 & 1 \\
\hline Haematuria & 6 & 4 & 1 & 1 & 0 & 3 \\
\hline Other GU & 4 & 0 & - & - & - & 0 \\
\hline Diarrhoea & 10 & 0 & - & - & - & 0 \\
\hline Abd pain/dyspepsia & 26 & 6 & 4 & 2 & 2 & 2 \\
\hline Malaria & 30 & 3 & 1 & 1 & 0 & 1 \\
\hline Infections & 23 & 1 & 0 & 0 & 0 & 2 \\
\hline Skin conditions & 14 & 0 & - & - & - & 0 \\
\hline Miscellaneous medical & 25 & 4 & 2 & 1 & 1 & 1 \\
\hline Total & 151 & 22 & 12 & 7 & 5 & 10 \\
\hline
\end{tabular}

Value of the LE test in screening for urethral infection

Twenty two FVU specimens from the outpatients (15\%) were positive by the LE test. We isolated $N$ gonorrhoeae from two of these 22 patients, and detected chlamydial antigen in the urine of five. A further five had NSU, defined as a PMN count of $\geqslant 5$ in the absence of these two agents. The positive predictive value of the LE test was $32 \%(7 / 22)$ for the detection of agents causing urethritis and $55 \%$ $(12 / 22)$ when allowing for NSU cases. The PMN count missed one case of gonorrhoea and two cases of chlamydial infection.

Because, for ethical reasons, we were not able to take urethral swabs from general medical outpatients with negative LE tests, it is not possible to calculate the sensitivity or specificity of the LE test for the detection of urethral infection in this group. For the same reason, only minimal estimates of prevalence can be given. The prevalences of $N$ gonorrhoeae, $C$ trachomatis and NSU were at least $1.3 \%$ $(2 / 151), 3 \cdot 3 \%(5 / 151)$ and $3 \cdot 3 \%(5 / 151)$ respectively, so that at least $8 \%(12 / 151)$ had urethral infection.

\section{FVU for the diagnosis of chlamydial infection}

Chlamydial antigen was detected in the urine of five general outpatients; in only two of these was it also detected in the urethral swab. None of 22 subjects tested had a positive swab result and a negative urine.

\section{Village residents}

Of 42 subjects seven (17\%) reported urethral symptoms; two of these had a urethral discharge $(5 \%)$. Twenty three $(55 \%)$ gave a past history of haematuria but only one complained of it at the time of the survey. The prevalence of LE positivity was $13 / 42$ (31\%).

\section{The effect of urinary schistosomiasis on the LE test}

The prevalence of Schistosoma haematobium infection was $2 / 55$ in the STD group (3.6\%), $10 / 151$ in the outpatient group (7\%) and $6 / 42$ in the village $(14 \%)$. Of the 18 subjects in whom $S$ haematobium was detected nine had a positive LE test (50\%), but three had concomitant gonorrhoea (two STD patients and one outpatient). Considering only the general medical outpatients and villagers, the prevalence of LE positivity was $7 / 16$ in those with $S$ haematobium (44\%) and 28/177 in those without (16\%); (Mantel-Haenszel test adjusting for patient group gives $\chi^{2}=4.42, p=0.04$ ). It was not possible to distinguish between schistosomiasis and urethritis by the level of positivity of the LE test or the presence of haematuria.

\section{Discussion}

The results of this study indicate that the LE test performed on a FVU specimen is a sensitive indicator of $C$ trachomatis or $N$ gonorrhoeae infection of the urethra in African males. Similar results were found in studies conducted in other settings in Europe and the 
$\mathrm{USA}^{14-18}$ : sensitivities in these studies ranged from $72 \%$ to $100 \%$ (our value $96 \%$ ), and specificities from $83 \%$ to $100 \%$ (our value $35 \%)$. The high sensitivity of the test suggests that this is an excellent screening method for identifying those patients who should be investigated for $N$ gonorrhoeae and $C$ trachomatis.

As a screening test for urethral infection, the LE test in our study gave a sensitivity of $94 \%$ and a specificity of $53 \%$. The apparently low specificity, or the high false positive rate, of the LE test in this study might be attributed to several factors. Generally recognised are: the limitations of the microscopic method for the diagnosis of urethritis (up to $35 \%$ false negative results according to some authors ${ }^{17}$ ), and the limitations of the diagnostic methods for $C$ trachomatis and $N$ gonorrhoeae. ${ }^{19}$ More specifically in our study, the frequency of selfmedication with antibiotics prior to consultation among STD patients was high $(42 \%)$ and might have decreased the recovery rate of infectious organisms. Low grade urethritis may persist in many subjects following inadequate self-medication, as reflected by the high prevalence of NSU cases. Finally, the LE test will be positive in cases of pyuria due to urinary tract infection as well as schistosomiasis.

Antigen detection tests based on direct immuno-fluorescence (DIF) or enzyme immunoassay (EIA)-especially those with confirmatory blocking assays-are increasingly recommended for the diagnosis of chlamydial infections. ${ }^{19-21} \mathrm{~A}$ number of studies have shown them to have a high specificity (above $98 \%$ ) and a high sensitivity (above $90 \%$ ). They have the considerable advantage that viable organisms are not required.

Because urethral swabbing is painful and may give rise to sampling errors, many authors have advocated the use of FVU for the diagnosis of chlamydial infection. ${ }^{19-22}$ Paul and $\mathrm{Caul}^{21}$ have advocated testing an early morning specimen because it should contain more chlamydial antigen than a FVU sample collected "on the spot" as recommended by Chernesky et al. ${ }^{20}$ Thomas et al ${ }^{22}$ found that there was no difference in sensitivity between the two timings. All authors agree that there should be a long enough delay ( 2 to 4 hours) between the last void and the collection of the specimen. Our study showed concordant results between urine specimens and urethral swabs in the STD group.

In the Outpatient group only two of five patients with urine specimens positive for chlamydial antigen also had positive swabs. It is likely that asymptomatic patients harbour few chlamydial elementary bodies (EBs) and that passing urine first might have a "washout" effect. For symptomatic patients the number of EBs is probably greater and the order of sampling does not affect the result; however patients should be advised to hold their urine for at least two hours before being assayed. Using a similar enzyme immunoassay for detecting gonococcal antigen on urine sediment proved to be sensitive (90-93\%), and specific $(98-99 \%)$, when compared with cul- ture, in other hands. ${ }^{1523}$ The possibility of using the same specimen for diagnosing both organisms is very attractive but was not evaluated in our study.

The prevalence rates for $N$ gonorrhoeae and $C$ trachomatis among patients with urethral syndrome were $54 \%$ and $10 \%$ respectively. This correlates well with what is usually found in similar settings elsewhere in Africa ${ }^{10_{11}^{13}}$; it is possible that a higher prevalence of chlamydial infection might have been found if additional antigen detection tests, or isolation had been used in this study. Prevalence rates of gonorrhoea are usually lower in Europe and North America and rates of $12-15 \%$ for chlamydial infection among symptomatic patients are currently reported. ${ }^{151718}$

The prevalence rates of gonorrhoea and chlamydial infection were at least $1.3 \%$ and $3.3 \%$ respectively among general medical outpatients. Rates of $2 \%$ to $5 \%$ of asymptomatic gonorrhoea are reported in North America among male adolescents ${ }^{1518}$ and asymptomatic antenatal clinic attenders in Ghana and The Gambia. ${ }^{1012}$ Higher rates $(5-10 \%)$ of asymptomatic chlamydial infection are quoted in the European and American literature. ${ }^{1518}$ It must be pointed out that these data apply to urban, adolescent populations, obtained during special clinic-based or prison-based surveys. These are obviously "high-risk" populations in terms of sexual exposure. We are not aware of any published data on the prevalence of asymptomatic chlamydial infection in African males. The literature indicates prevalence rates of $5 \%-10 \%$ among African female groups (for example, antenatal clinic attenders)..$^{9-12}$ It is likely to be lower among asymptomatic males, thus reproducing the pattern found in the more developed countries. $^{15-18}$

It is clear that the success of any STD control programme depends critically on the prevalence of asymptomatic infections. Efforts to decrease the spread of $N$ gonorrhoeae or $C$ trachomatis have focussed on screening asymp tomatic females in Africa ${ }^{112}$ but much less attention has been paid to asymptomatic male infections. In this study the LE test was able to detect cases of gonococcal or chlamydial urethritis among patients without STD symptoms. It also correctly identified symptomatic patients, even those with vague complaints or uncertain signs. Used in the context of an STD screening programme, in a general male population, the LE test can be an important epidemiological tool. The same dipstick, with its erythrocyte reagent strip, can also identify $S$ haematobium infected patients, which is an additional benefit in an endemic area. However, it is not possible to distinguish subjects with urethritis and schistosomiasis from those with schistosomiasis alone.

We found that the use of the LE test for screening asymptomatic males was cost-effective compared with screening all asymptomatic males by urethral cultures. It was possible to reduce the number of cultures and IDEIA tests by $82 \%$ (from 193 to 35 ). In a large scale STD screening programme this means important 
savings in terms of cost, labour and unnecessary discomfort for the subjects. Furthermore the test is cheap (a few US cents), very easily performed and reasonably objective for use in the field.

We conclude that urine testing can play an important role in STD control programmes in developing countries. It is possible that urine can be used both for screening (LE test) and diagnosis (antigen detection) using the same urine specimen. Gonococcal antigen detection has shown promise in other hands and we have demonstrated that chlamydial antigen detection by IDEIA is also a reliable test. The frequency of chlamydial infection detected only in urine specimens of asymptomatic patients highlights the need to use these specimens, in order to know the exact prevalence of such infections.

This work was supported in part by grants from the EC AIDS Task Force and the Overseas Development Administration of the UK. P Mayaud was the recipient of a grant from the Institut Beecham, Paris. Dipstick tests (Nephur-Test + Leuco) were Beecham, Paris. Dipstick tests (Nephur-Test + Leuco) were provided by courtesy of the Laboratoires Boehringer-MannMumtaz for useful advice at different stages of the project.

1 Piot P, Plummer FA, Mhalu FS, et al. AIDS: an international perspective. Science 1988;239:573-9.

Chin J. Global estimates of AIDS cases and HIV infection: 1990. AIDS 1990;4(suppl 1):S277-83.

3 Quinn TC. The epidemiology of the acquired immune deficiency syndrome and the immunological responses to the human immunodeficiency virus. Current Opinion in Immunology 1989;1:502-12.

4 Simonsen JN, Cameron DW, Gakinya $\mathrm{MN}$, et al. Human immunodeficiency virus infection among men with sexually transmitted diseases. Experience from a center Africa. $N$ Engl ₹ Med 1988;319:274-7.

5 Cameron DW, Simonsen JN, D'Costa LJ et al. Female to male transmission of human immunodeficiency virus type 1: risk factors for seroconversion in men. Lancet 1989 ii:403-7.

6 Plummer FA, Simonsen JN, Cameron DW, et al. Co-factors in male-female sexual transmission of human immunodeficiency virus type 1. F Infect Dis 1991;163:233-9.

7 Mertens TE, Hayes RJ, Smith PG. Epidemiological methods to study the interaction between HIV infection and other sexually transmitted diseases. AIDS 1990;4:57-65.

8 Pepin J, Plummer FA, Brunham RD, et al. The interaction of HIV infection and other sexually transmitted diseases: an opportunity for intervention. AIDS 1989;3:3-9.

an opportunity for intervention. AIDS 1989;3:3-9. AIDS. Postgraduate Doctor Middle East, 1991;13:128-30.

10 Mabey DCW, Whittle HC. Genital and neonatal chlamydial infection in a trachoma endemic area. Lancet 1982 ii:300-1.

11 Leclerc A, Frost E, Collet M, Goeman J, Bedjabaga L. Urogenital Chlamydia trachomatis in Gabon: an unrecog nised epidemic. Genitourin Med 1988;64:308-11.

12 Bentsi C, Klufio A, Perine PL, et al. Genital infections with Chlamydia trachomatis and Neisseria gonorrhoeae in Ghanian women. Genitourin Med 1985;61:48-50.

13 Arya OP. Sexually transmitted diseases: In: Robinson D, ed. Epidemiology and the Community Control of Disease in Warm Climate Countries. Edinburgh: Churchill Livingstone, 1985:348-59.

14 Perera SAB, Jones C, Srikantha V, et al. Leucocyte esterase test as rapid screen for non-gonococcal urethritis. Genitourin Med 1987;63:380-3.

15 Shafer MA, Schachter J, Moscicki AB, et al. Urinary leukocyte esterase screening test for asymptomatic chlamydial and gonococcal infections in males. $¥ A M A$ 1989;262:2562-6.

16 Sadof MD Woods MD, Emans SJ. Dipstick leukocyte esterase activity in first-catch urine specimens. $\mathscr{f} A M A$, 1987;258:1932-4.

17 Veeravahu M, Smyth RW, Clay JC. Detection of leukocyte esterase in urine: a new screening test for non-gonococcal urethritis compared with two microscopic methods. Sexually transmitted diseases, 1987;14:180-4.

18 O'Brien SF, Bell TA, Farrow JA. Use of a leukocyte esterase dipstick to detect Chlamydia trachomatis and Neisseria gonorrhoeae urethritis in asymptomatic adolescent male detainees. Am $\mathcal{F}$ Public Health 1988;78:1583-4.

19 Ridgway GL, Taylor-Robinson D. Current problems in microbiology. Chlamydial infections: which laboratory test? $f$ Clin Pathol 1991;44:1-5.

20 Chernesky M, Catrisciano S, Sellors J, et al. Detection of Chlamydia trachomatis antigens in urine as an alterntive to swabs and cultures. F Infect Dis 1990;161:124-6.

21 Paul ID, Caul EO. Evaluation of three Chlamydia trachomatis immunoassays with an unbiased, non-invasive chomatis immunoassays with an unbiased, non-in

22 Thomas BJ, Gilchrist C, Taylor-Robinson D. Simplification of procedures used to test urine samples for Chlamydia trachomatis. F Clin Pathol 1991;44:374-5.

23 Demetriou E, Sackett R, Welch DF, Kaplan DW. Evaluation of an Enzyme Immunoassay for detection of Neisseria gonorrhoeae in an adolescent population. $\mathcal{F} A M A, 1984$ 252:247-50 